\section{Letter: Relearning the 3 R's? Reinterpretation, recontact, and return of genetic variants}

The genetic policy world is currently ablaze with debates over the extent of clinician and researcher responsibilities to reinterpret variants and recontact patients and participants, to say nothing of obligations to return results and secondary findings.

The American College of Medical Genetics and Genomics (ACMG) fired the latest volley in December 2018 with its "Patient re-contact after revision of genomic test results: points to consider." The policy recognizes that laboratories, patients, and physicians (called "ordering providers") share responsibility for communication where the clinical meaning of genomic data changes (e.g., where variants are reinterpreted, the list of reported variants expands, or richer testing platforms are adopted). While the ordering provider is the primary coordinator of care, ultimate responsibility is left with the patient: "The ordering provider...cannot promise that recontact regarding a revised interpretation will occur unless the patient initiates the re-contact" (point 3). The ACMG recommends informed consent and pre- and posttest counseling be employed to clarify the limits of professional responsibility and the corollary obligations of the patient. In other words, consumer (patient) beware!

This stands in sharp contrast to the Position Statement of the American Society of Human Genetics (ASHG) on the duty to recontact in the research environment. ${ }^{2}$ Even though this Statement addresses the researcher-participant relationship, it "strongly recommends attempting to recontact participants to offer updated results if the reinterpretation is related to the phenotype under study or is reasonably expected to affect a research participant's medical management" (recommendation 1). On its surface, this would create duties for researchers and rights for participants that far surpass those clinicians have toward their patients under the ACMG Points to Consider. Recontact is also advised when reinterpretation is not expected to affect management but where the classification of a variant is corrected from or to pathogenic/likely pathogenic (recommendation 2). Fortunately, researchers are relieved from a possible duty to hunt for changes in variant interpretation (i.e., a duty to reanalyze) (recommendation 3 ). The duty to recontact is limited in time to the duration of the research funding (recommendation 4), and in substance to a "good faith effort" within the limits of existing constraints (recommendation 8). Both policies respect individual choice. While the right not to know is less controversial in the context of reinterpretation, it is still tricky to operationalize. The ACMG preserves a patient's right to decline recontact (point 1e). The ASHG considers a general consent to the return of results at the time of recruitment to imply consent to recontact for the same type of result (recommendation 10).

The ACMG and ASHG policies seem contradictory, and both deviate from traditional health professional and researcher duties, albeit in opposite directions. The two policies could not be further apart in spirit. The ACMG stance is medicolegal, appealing to the law and recommending exculpatory communication strategies. The ASHG position is aspirational and bioethical but would expand the hitherto fiduciary but circumscribed bounds of researcher obligations.

These policies are set against the ongoing, vociferous debate over the return of results that followed the release of the 2018 report by the US National Academies of Sciences, Engineering, and Medicine Returning individual research results to participants: Guidance for a new research paradigm. ${ }^{3}$ This report rightly places particular emphasis on enforceable, quality standards for research laboratories so as to achieve greater accuracy, validity, and reproducibility as a precursor to any return of results. The report was followed by lively interpretive debate over whether the Clinical Laboratory Improvement Amendments of 1988 prohibit research laboratories without CLIA accreditation from returning results. ${ }^{4-6}$

The original three R's - reading writing and 'rithmeticwere basic to education. Skills to be learned in geneticsreinterpretation, recontact, and return-seem to be avoided by the ACMG and exaggerated by the ASHG. The 2018 Statement of the European Society of Human Genetics (ESHG) on recontact in the clinic raises a fourth " $R$ " discounted in the American debate: resource constraints. ${ }^{7}$ Indeed, mounting professional obligations and associated costs may restrain the uptake of genomic research into the clinic especially in countries with health-care systems based on universal and equitable access. The ESHG eschews a positive obligation to recontact ("there is currently no duty to do so") and recommends that clinical genetics services make determinations based on "the best interests of the patient/ family" (recommendation 1).

Improving patient care is the ultimate consideration for any clinical or research guideline. Simply diverting the information deluge from physicians to patients is unlikely to serve their interests, or engender their trust in genomic medicine. The ASHG policy is rightly concerned about the welfare of research participants, and fairness between them. But pushing these bioethical concerns too far can burden research and slow the uptake of genomic medicine-to the detriment of future patients.

Can a more consistent and coherent genetic variant pathway with the necessary communication feedback loops 
be designed for the new 4 R's? Back to basics begins with the laboratory. Quality and safety are the foundations of responsible professionalism and respect for the rights and possible future duties of research participants and patients. Treating patients and research participants as true partners demands no less if we are to build a true "learning" health system.

\section{DISCLOSURE}

The authors declare no conflicts of interest.

Publisher's note: Springer Nature remains neutral with regard to jurisdictional claims in published maps and institutional affiliations.

Bartha Maria Knoppers, $P h D^{1}$, Adrian Thorogood, BCL, LLB ${ }^{1}$ and $M a^{\prime} n$ H. Zawati, DCL ${ }^{1}$

${ }^{1}$ Centre of Genomics and Policy, McGill University, Montreal, QC, Canada Correspondence: Bartha Maria Knoppers (bartha.knoppers@mcgill.ca)

\section{REFERENCES}

1. David $K L$, et al. Patient re-contact after revision of genomic test results: points to consider-a statement of the American College of Medical Genetics and Genomics (ACMG). Genet Med. 2018; https://www. nature. com/articles/s41436-018-0391-z [Epub ahead of print].

2. Bombard $Y$, et al. ASHG Position Statement: The Responsibility to Recontact Research Participants after Reinterpretation of Genetic and Genomic Research Results. AJHG. 2019;104:578-595.

3. National Academies of Sciences, Engineering, and Medicine. Returning individual research results to participants: guidance for a new research paradigm. Washington, DC: The National Academies Press. 2018; https://doi.org/10.17226/25094

4. Wolf SM, Evans BJ. Return of results and data to study participants. Science. 2018;362:159-160.

5. Botkin JR, et al. Standardizing return of participant results. Science. 2018;362:759-760.

6. Wolf SM, Evans BJ. Defending the return of results and data. Science. 2018;362:1255-1256.

7. Carrieri D, et al. Recontacting patients in clinical genetics services: recommendations of the European Society of Human Genetics. Eur J Hum Genet. 2019;27:169-182.

Advance online publication 11 April 2019. doi:10.1038/s41436-019-0494-1 